\title{
BMC Biochemistry reviewer acknowledgement, 2014
}

Guangde Tu

\section{Contributing reviewers}

The editors of BMC Biochemistry would like to thank all our reviewers who have contributed their time to the journal in Volume 15 (2014).

\begin{tabular}{|c|c|c|}
\hline $\begin{array}{l}\text { Ana I. Azuaga } \\
\text { Spain }\end{array}$ & $\begin{array}{l}\text { Laurent Desaubry } \\
\text { France }\end{array}$ & $\begin{array}{l}\text { Marina Guizzetti } \\
\text { USA }\end{array}$ \\
\hline $\begin{array}{l}\text { Nafisa Balasinor } \\
\text { India }\end{array}$ & $\begin{array}{l}\text { Maria Grazia Esposito } \\
\text { Italy }\end{array}$ & $\begin{array}{l}\text { Bassem Hassan } \\
\text { Belgium }\end{array}$ \\
\hline $\begin{array}{l}\text { David Bentley } \\
\text { USA }\end{array}$ & $\begin{array}{l}\text { Daniela Fietz } \\
\text { Germany }\end{array}$ & $\begin{array}{l}\text { Harald Herrmann } \\
\text { Germany }\end{array}$ \\
\hline $\begin{array}{l}\text { Tadayoshi Bessho } \\
\text { USA }\end{array}$ & $\begin{array}{l}\text { Theodore Fotsis } \\
\text { Greece }\end{array}$ & $\begin{array}{l}\text { Michal Hetman } \\
\text { USA }\end{array}$ \\
\hline $\begin{array}{l}\text { Volker Briken } \\
\text { USA }\end{array}$ & $\begin{array}{l}\text { Nicole Francis } \\
\text { Canada }\end{array}$ & $\begin{array}{l}\text { Jim Horn } \\
\text { USA }\end{array}$ \\
\hline $\begin{array}{l}\text { Ana Busturia } \\
\text { Spain }\end{array}$ & $\begin{array}{l}\text { Octavio Franco } \\
\text { Brazil }\end{array}$ & $\begin{array}{l}\text { Jianghui Hou } \\
\text { USA }\end{array}$ \\
\hline $\begin{array}{l}\text { Francisco Campos } \\
\text { Brazil }\end{array}$ & $\begin{array}{l}\text { Christian Frezza } \\
\text { United Kingdom }\end{array}$ & $\begin{array}{l}\text { Thomas Hurley } \\
\text { USA }\end{array}$ \\
\hline $\begin{array}{l}\text { Guifang Cao } \\
\text { China }\end{array}$ & $\begin{array}{l}\text { Suthat Fucharoen } \\
\text { Thailand }\end{array}$ & $\begin{array}{l}\text { Ibtessam Hussein } \\
\text { Saudi Arabia }\end{array}$ \\
\hline $\begin{array}{l}\text { MI-LA Cho } \\
\text { South Korea }\end{array}$ & $\begin{array}{l}\text { Ramesh Ganju } \\
\text { USA }\end{array}$ & $\begin{array}{l}\text { Kazumi Ishidoh } \\
\text { Japan }\end{array}$ \\
\hline $\begin{array}{l}\text { Mark Chong } \\
\text { Australia }\end{array}$ & $\begin{array}{l}\text { Jan Gettemans } \\
\text { Belgium }\end{array}$ & $\begin{array}{l}\text { Atsuo Iwasawa } \\
\text { Japan }\end{array}$ \\
\hline $\begin{array}{l}\text { Jane Elizabeth Collins } \\
\text { United Kingdom }\end{array}$ & $\begin{array}{l}\text { Celia Goulding } \\
\text { USA }\end{array}$ & $\begin{array}{l}\text { Marek Jankoiwski } \\
\text { Canada }\end{array}$ \\
\hline $\begin{array}{l}\text { Yu-Sheng Cong } \\
\text { China }\end{array}$ & $\begin{array}{l}\text { Matt Guille } \\
\text { United Kingdom }\end{array}$ & $\begin{array}{l}\text { Xinhua Ji } \\
\text { USA }\end{array}$ \\
\hline $\begin{array}{l}\text { Kalavathi Dasuri } \\
\text { USA }\end{array}$ & $\begin{array}{l}\text { Hervé Guillou } \\
\text { France }\end{array}$ & $\begin{array}{l}\text { Anne Katherine Jones } \\
\text { USA }\end{array}$ \\
\hline \multicolumn{3}{|c|}{$\begin{array}{l}\text { Correspondence: guangde.tu@biomedcentral.com } \\
\text { Room 1006-1007 Financial Plaza, No. } 333 \text { Jiujiang Road, Huangpu District, } \\
\text { Shanghai 200001, China }\end{array}$} \\
\hline
\end{tabular}


Bavesh Davandra Kana

South Africa

Paul King

USA

Essam Kotb

Egypt

Adam Krieg

USA

Isabelle Landrieu

France

Konstantinos Lefkimmiatis

United Kingdom

Christina Leslie

USA

Jie Li

USA

Stefan Lichtenthaler

Germany

Jing-Jer Lin

Taiwan

Peter Lindblad

Sweden

Dan Lindholm

Finland

ZuFu Lu

Australia

Erich Mackow

USA

Lee Macomber

USA

Sandro Marana

Brazil

Neil McDonald

United Kingdom

John McLenithan

USA

Rod Merrill

Canada

Joris Messens

Belgium

John Mieyal

USA

Isabelle Mus-Veteau

France
Masato Nakai

Japan

Fengyun Ni

USA

Ken Okazaki

Japan

Angela Pennacchio

Italy

Giulia Piaggio

Italy

Miles Pufall

USA

Pradman Qasba

USA

Jose Luis R. Arrondo

Spain

Arumugam Rajavelu

India

Francois Robert

Canada

Christoph Romanin

Austria

George Russev

Bulgaria

Avneesh Saini

USA

Roger Sandwick

USA

Neelam Sangwan

India

Devanand Sarkar

USA

Jitendra Saxena

India

Gerd Schmitz

Germany

Claudia Scholl

Germany

Tobias Schuerholz

Germany

Benjamin Schulz

Australia

Dominik Schwudke

Germany
Jose Segovia

Mexico

Olli Silvennoinen

Finland

Alexander Skurat

USA

Guanfang Su

China

Shuo Sun

USA

Avadhesha Surolia

India

Sanjay Swarup

Singapore

Liskin Swint-Kruse

USA

Daniel Taillandier

France

Yuichiro Takanami

Japan

Tien-Chye Tan

Sweden

Winston Thompson

USA

Nguyen Trong Tue

Viet Nam

Kouhei Tsumoto

Japan

Takafumi Uchida

Japan

John Ussher

Canada

Martin van der Laan

Germany

Cornelis Van Noorden

Netherlands

Joseph Vasselli

USA

Roberto Vazquez-Padron

USA

Jan Willem Voncken

Netherlands

Geerten W. Vuister

United Kingdom 
Chong Wang

China

Oliver Weichenrieder

Germany

Catherine Winder

United Kingdom

Dieter Wolf

USA
Kinnosuke Yahiro

Japan

Zhaogang Yang

USA

Lennart Zabeau

Belgium

Jianye Zang

China
Haili Zhang

USA

Guoqing Zhang

USA 